\title{
BMJ Open Work stress and oral conditions: a systematic review of observational studies
}

\author{
Yukihiro Sato (D), Yasuaki Saijo (D) , Eiji Yoshioka
}

To cite: Sato Y, Saijo Y, Yoshioka E. Work stress and oral conditions: a systematic review of observational studies. BMJ Open 2021;11:e046532. doi:10.1136/ bmjopen-2020-046532

- Prepublication history and additional supplemental material for this paper are available online. To view these files, please visit the journal online (http://dx.doi.org/10.1136/ bmjopen-2020-046532).

Received 04 November 2020 Accepted 03 May 2021
Check for updates

(C) Author(s) (or their employer(s)) 2021. Re-use permitted under CC BY-NC. No commercial re-use. See rights and permissions. Published by BMJ.

Division of Public Health and Epidemiology, Department of Social Medicine, Asahikawa Medical University, Asahikawa Hokkaido, Japan

Correspondence to

Dr Yukihiro Sato; ys@epid.work

\begin{abstract}
Objectives Although psychological stress is a risk factor for oral diseases, there seems to be no review on work stress. This study aimed to review the evidence on the association between work stress and oral conditions, including dental caries, periodontal status and tooth loss. Design A systematic review of published observational studies.
\end{abstract}

Data sources A systematic literature search was conducted in PubMed and Scopus databases on 12 August 2020.

Study selection Articles were screened based on the following inclusion criteria: published after 1966; in English only; epidemiological studies on humans (except case studies, reviews, letters, commentaries and editorials); and examined the association of work stress with dental caries, periodontal status and tooth loss.

Data extraction Data were extracted from eligible studies. A quality assessment was conducted using the Quality Assessment Tool for Observational Cohort and Cross-Sectional Studies.

Results 0 402 articles identified, 11 met the inclusion criteria, and 1 study assessed the association of work stress with dental caries and periodontal status. Of 11 studies, 1 reported a non-significant association between work stress and dental caries; 8 of 9 studies reported a significant association between work stress and worse periodontal status; and 1 of 2 studies reported a significant association between work stress and tooth loss. Nine of 11 studies were cross-sectional, while the remaining 2 studies had unclear methodology. Only two studies were sufficiently adjusted for potential confounders. Eight studies assessed work stress but did not use the current major measures. Three studies were rated as fair, while eight studies had poor quality.

Conclusions There is a lack of evidence on the association of work stress with dental caries and tooth loss. Eight studies suggested potential associations between periodontal status and work stress. Cohort studies using the major work stress measures and adjusting for the potential confounders are needed.

\section{INTRODUCTION}

Oral diseases, such as dental caries and periodontal disease, are a major health concern worldwide. The Global Burden of Disease Study has estimated that 2.3 billion individuals had untreated dental caries, 796 million had severe periodontal disease and 267 million had a complete loss of natural teeth
Strengths and limitations of this study

- This is the first systematic review to evaluate and summarise the literature on the association between work stress and oral conditions, including dental caries, periodontal status and tooth loss.

- This systematic review provides a comprehensive insight into the quality of the included papers.

- The systematic literature search, screening and quality assessments were conducted by only one investigator.

- A meta-analysis could not be conducted because of the heterogeneity of work stress measures and outcome definitions.

in 2017. ${ }^{1}$ Dental caries is the destruction of dental hard tissues in the crowns and roots of the teeth. ${ }^{2}$ Periodontal diseases are chronic inflammatory conditions with disorders of the tissues surrounding and supporting the teeth. ${ }^{3}$ Tooth loss is mainly the consequence of dental caries and periodontal disease. ${ }^{23}$ Because oral diseases result in severe toothache and eating, sleeping, and communication disabilities, ${ }^{45}$ poor oral conditions can restrict work performance ${ }^{45}$ and create a significant economic burden. ${ }^{6}$ Indeed, work productivity loss due to oral conditions is estimated at US\$187.61 billion annually. ${ }^{6}$ The necessity of preventing oral diseases for working adults is highlighted.

Since the 1990s, rapid changes in the global economy and the diverse markets have occurred, and psychological workplace stress has become more prevalent and severe, especially among industrialised countries. ${ }^{7}$ Indeed, Kivimäki et al reported a $15 \%$ prevalence of job strain measured using job content and demand control questionnaires from 13 European cohorts' data (1985-2006). ${ }^{8}$ Besides, work stress can have profound effects on health. There is accumulating evidence of the risk of work stress on cancer, cardiovascular diseases, diabetes and depression. ${ }^{9} 10$ Béjean and Sultan-Taieb estimated that the work-related stress costs due to illnesses could range between $€ 1167$ million and $€ 1975$ 
million in France in 2000. ${ }^{11}$ Work stress affects workers' health and productivity.

Psychological stress is recognised as a risk factor for dental caries and periodontal diseases. Psychological stress is related to oral diseases through immune system dysfunction, increased stress hormones, cariogenic bacterial counts and poor oral health behaviours. ${ }^{12}{ }^{13}$ Work stress is strongly linked with psychological and physical health. ${ }^{9} 10$ Previous systematic reviews suggested potential associations of psychological stress with dental caries and periodontitis. ${ }^{14} 15$ However, there seems to be no review on the association between work stress and oral diseases. Today, work stress has become an increasingly serious problem. Besides, the number of women in the workforce and dual-earner families has been increasing. ${ }^{16}$ A wide range of populations can suffer the risk of oral diseases from exposure to work stress. Thus, the aim of this systematic review was to evaluate and summarise the literature on the association between work stress and oral conditions, including dental caries, periodontal status and tooth loss. We set the following review question: Is work stress associated with dental caries, periodontal status and tooth loss among working adults?

\section{METHODS}

The reporting of this systematic review conforms to the Preferred Reporting Items for Systematic Reviews and Meta-Analyses guidelines. ${ }^{17}{ }^{18}$ We also followed the Conducting Systematic Reviews and Meta-Analyses of Observational Studies of Etiology guidance ${ }^{19}$ and the reporting of Meta-analysis Of Observational Studies in Epidemiology. ${ }^{20}$ The protocol of this systematic review was not registered.

\section{Eligibility criteria}

Published studies were eligible if they: (1) were published in English; (2) were epidemiological studies on humans (except case studies, reviews, letters, commentaries and editorials); and (3) examined the association of work stress with dental caries, periodontal status and tooth loss.

\section{Information sources and searches}

On 12 August 2020, we identified potentially relevant published studies in PubMed (1966-12 August 2020) and Scopus (1966-12 August 2020) databases. As PubMed and Scopus have only data back to 1966, we focused on articles published after 1966. We used the following script to obtain a wide range of literature: ("job strain" OR "effort reward") AND (dental OR oral); ("job stress" OR "work stress" OR "occupational stress") AND (dental OR oral). The details of the search strategies for each database are shown in online supplemental table 1 . Besides, we manually hand-searched for potentially suitable studies through the reference lists of identified articles and Google Scholar. After excluding duplicate articles, one author (YSato) assessed the titles and abstracts according to the aforementioned criteria. Then, eligible studies were selected for the full-text review.

\section{Data extraction}

One author (YSato) extracted the following information from each eligible study: (1) name of the first author; (2) study design; (3) study location (country); (4) number of participants and work-related characteristics; (5) exposure and its measurements; (6) outcome and its measurements; (7) age range and proportion of women; (8) covariates included in the adjusted models and (9) the main results. The results were shown in table 1 .

\section{Quality assessment}

We used the Quality Assessment Tool for Observational Cohort and Cross-Sectional Studies to assess the quality of included studies. ${ }^{21}$ This tool includes 14 questions for evaluating the internal validity of a study and these questions are documented in the footnote of table 2. For each question, one author (YSato) rated them as yes, no or other (including cannot determine, not reported and not applicable). The overall quality rating for the study was regarded as good if all the domains were assessed favourably.

\section{Synthesis of results}

A meta-analysis could not be conducted because of the heterogeneity of work stress measures and outcome definitions.

\section{Patient and public involvement}

No patient involved.

\section{RESULTS}

Figure 1 presents the flow diagram of information through the phases of the systematic review. Of the 402 articles identified in PubMed and Scopus databases, 129 duplicated articles were removed, the titles and abstracts of 273 were screened, and 11 met the eligibility criteria. Three more articles identified through reference lists and hand-search were added. One article was identified by a hand-search using Google Scholar, ${ }^{22}$ one was from a reference list $^{23}$ and the third was an article ${ }^{24}$ plagiarised by a retraction paper. Because the article $^{24}$ which was plagiarised by the retracted one was published officially and has not been retracted, it was included in our references. After full-text assessments of 14 articles, 3 were excluded due to retraction $(n=1)$ and the use of composite outcomes including dental caries and periodontal status $(\mathrm{n}=2) .{ }^{25}{ }^{26}$ Finally, 11 articles were included in this systematic review. ${ }^{22-24} 27-34$

\section{Study characteristics of individual studies}

Table 1 shows the 12 summaries from the 11 studies. One of 11 studies reported on dental caries and periodontal status, ${ }^{27} 8$ reported on periodontal status ${ }^{22-24}{ }^{28-32}$ and 2 reported on tooth loss. ${ }^{334}$ Three studies were conducted in Japan, ${ }^{3032-34}$ two in India, ${ }^{241}$ and one each in the UK, ${ }^{28}$ 


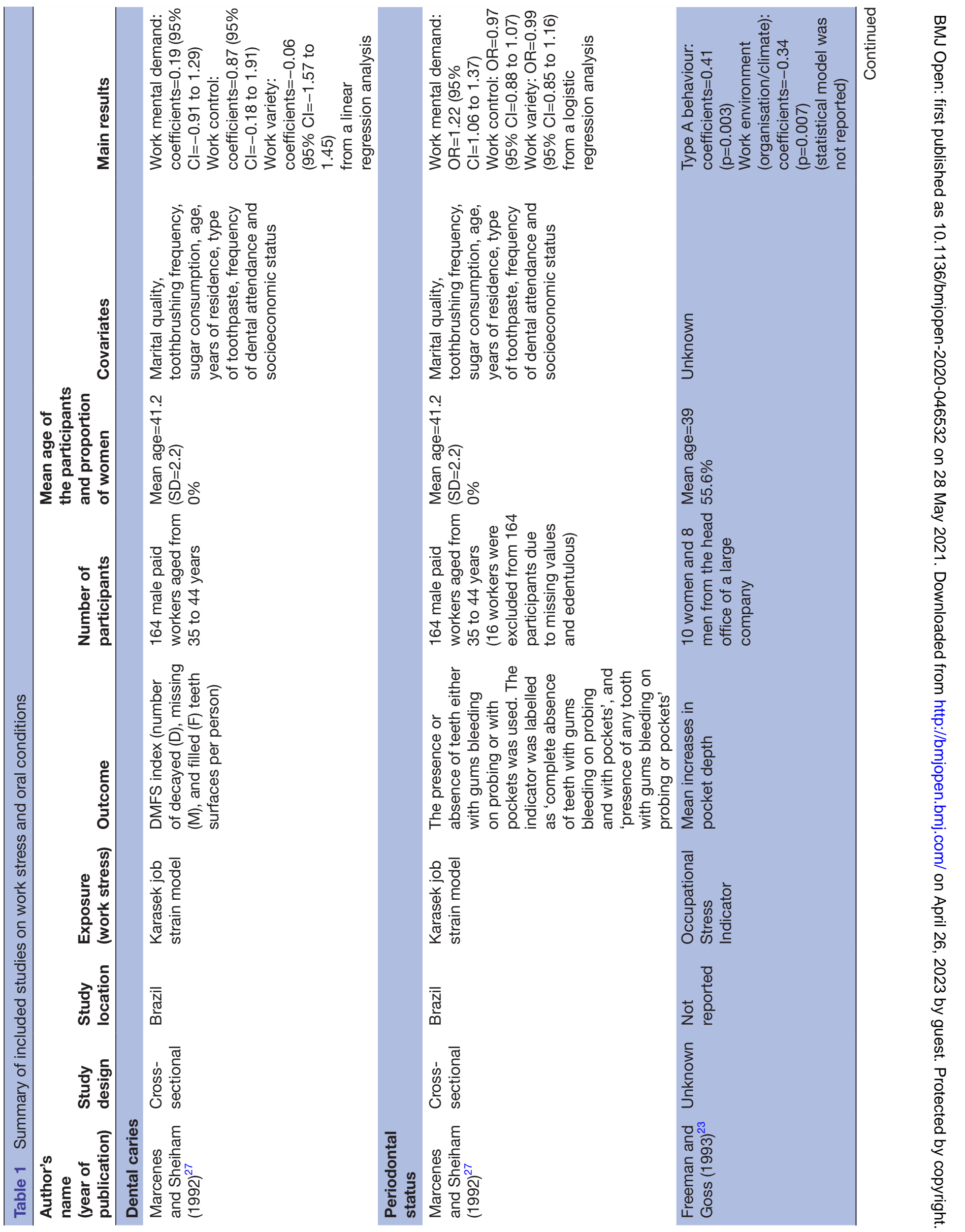




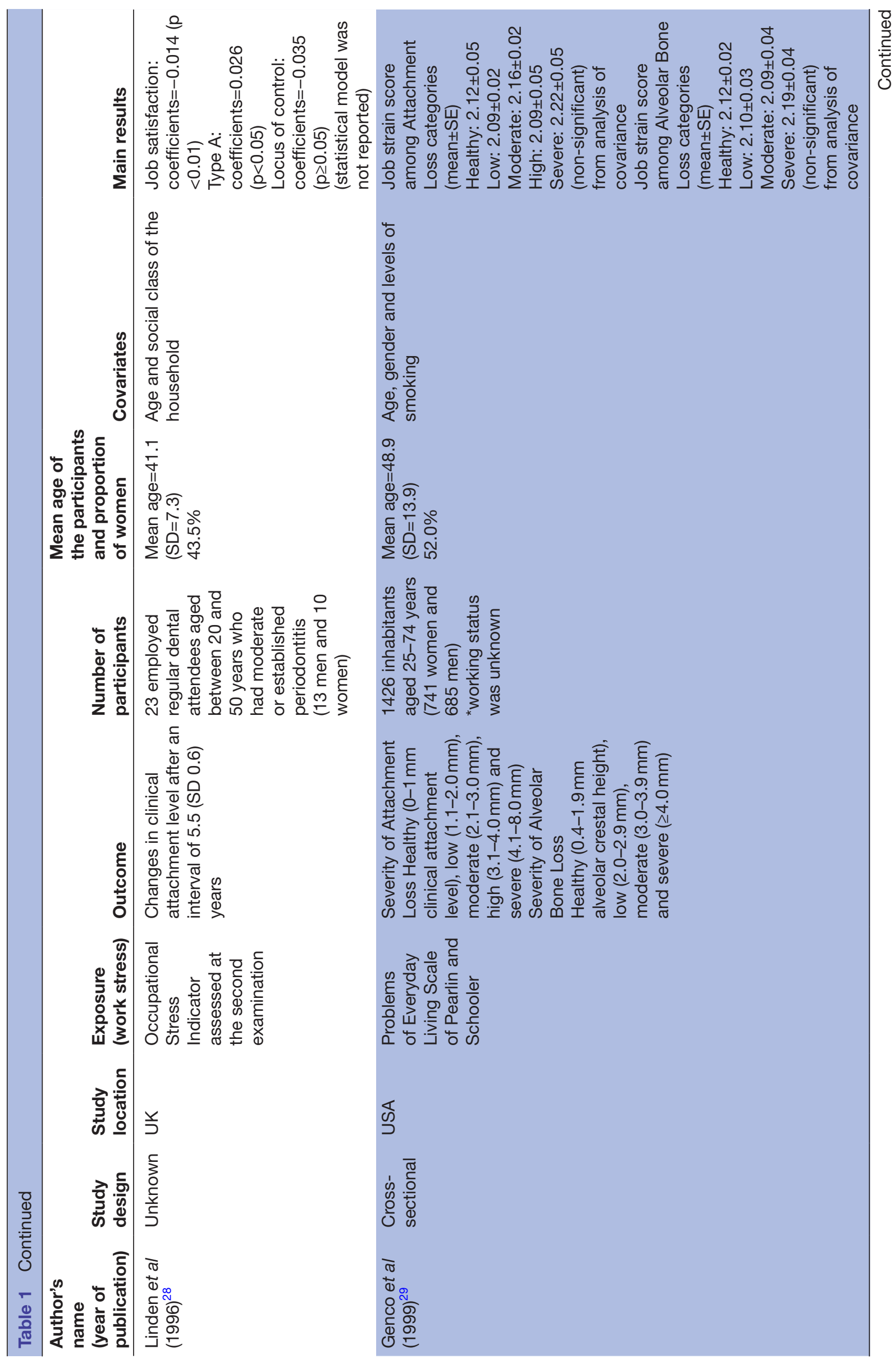




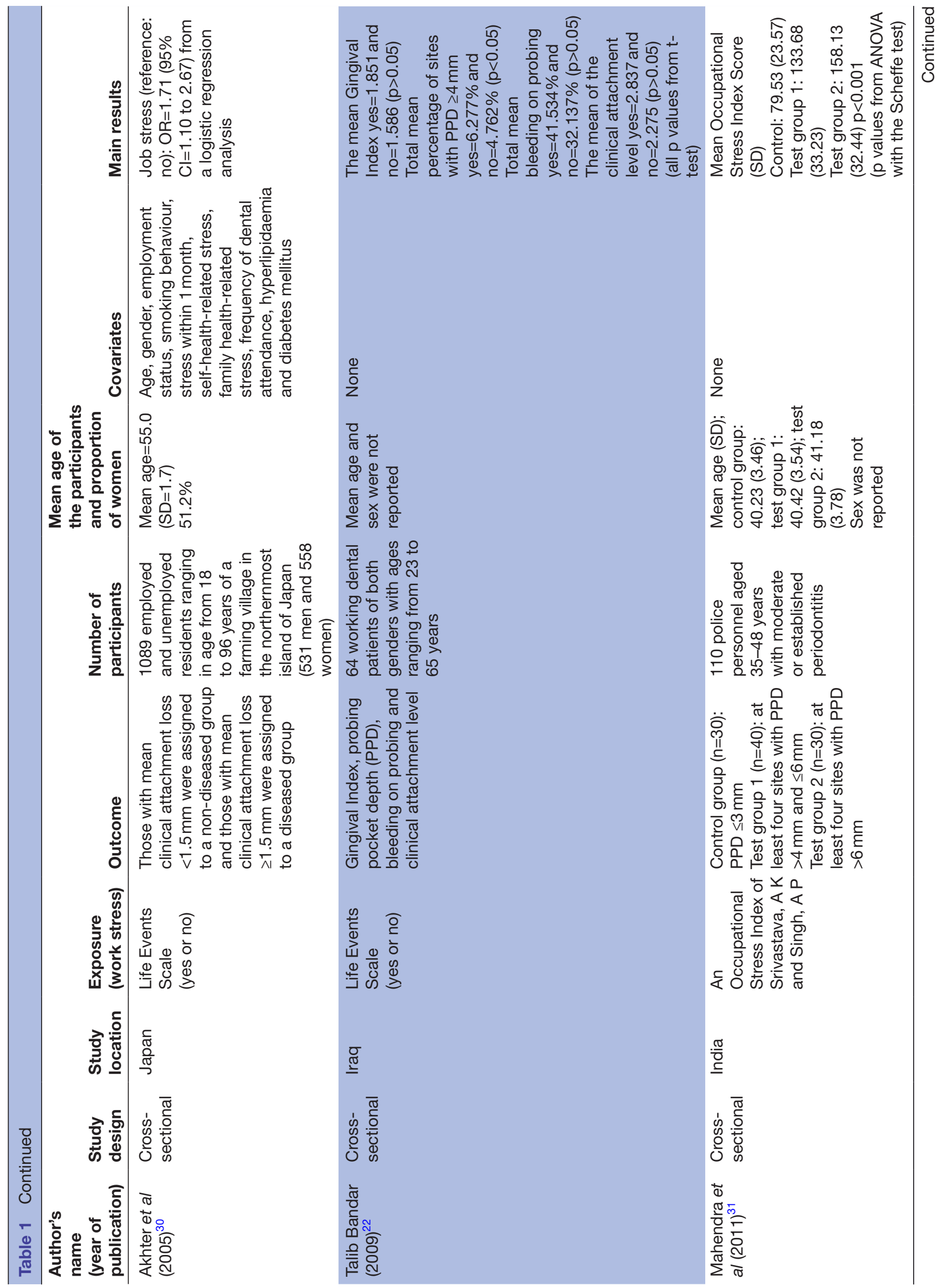




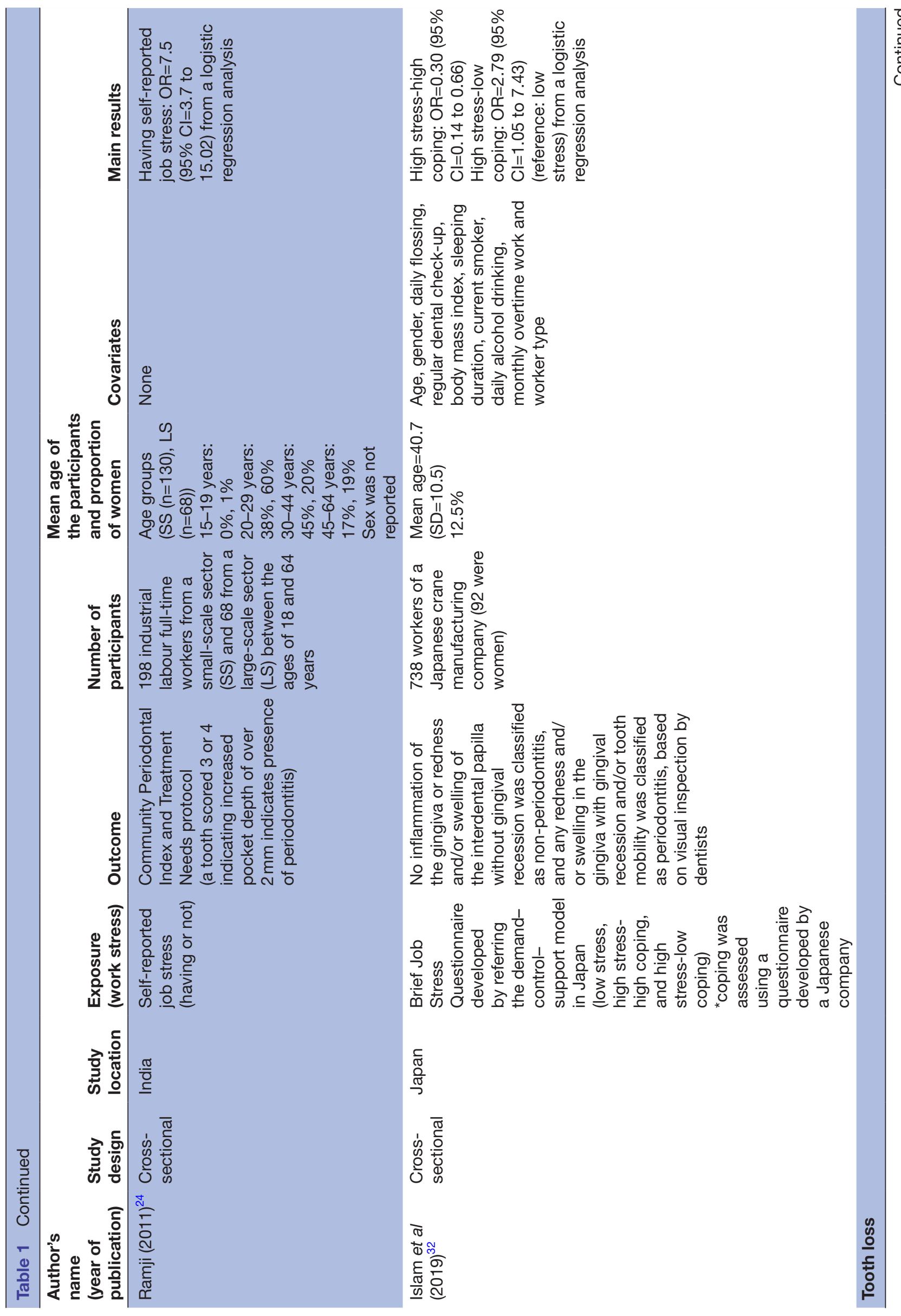




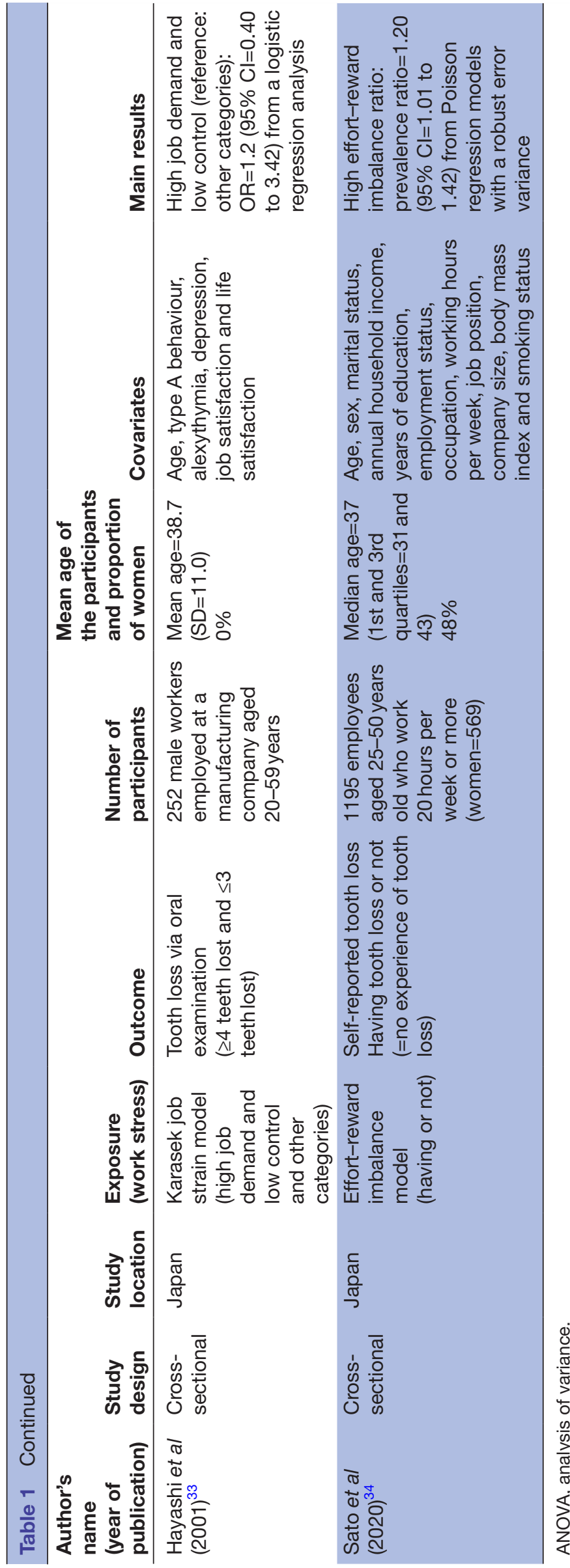


Table 2 Quality assessment of included studies

\begin{tabular}{|c|c|c|c|c|c|c|c|c|c|c|c|c|c|c|c|}
\hline & Q1 & Q2 & Q3 & Q4 & Q5 & Q6 & Q7 & Q8 & Q9 & Q10 & Q11 & Q12 & Q13 & Q14 & $\begin{array}{l}\text { Quality rating (good, } \\
\text { fair or poor) }\end{array}$ \\
\hline $\begin{array}{l}\text { Marcenes and } \\
\text { Sheiham }^{27}\end{array}$ & Yes & Yes & NR & Yes & Yes & No & No & Yes & Yes & No & Yes & Yes & NA & Yes & Fair \\
\hline Linden et $a^{28}$ & Yes & Yes & NR & Yes & Yes & No & No & Yes & Yes & No & Yes & Yes & NA & No & Poor \\
\hline Akhter et $a^{30}$ & Yes & Yes & NR & No & Yes & No & No & NA & No & No & Yes & Yes & NA & No & Poor \\
\hline Talib Bandar 22 & Yes & Yes & NR & No & No & No & No & NA & No & No & Yes & Yes & NA & No & Poor \\
\hline Mahendra et $a l^{31}$ & Yes & Yes & NR & Yes & Yes & No & No & NA & Yes & No & Yes & Yes & NA & No & Poor \\
\hline Ramji $^{24}$ & Yes & Yes & No & Yes & Yes & No & No & NA & No & No & Yes & Yes & NA & No & Poor \\
\hline Islam et $\left.a\right|^{32}$ & Yes & Yes & NR & Yes & Yes & No & No & NA & No & No & Yes & Yes & NA & No & Poor \\
\hline
\end{tabular}

Q1. Was the research question or objective in this paper clearly stated?

Q2. Was the study population clearly specified and defined?

Q3. Was the participation rate of eligible persons at least $50 \%$ ?

Q4. Were all the subjects selected or recruited from the same or similar populations (including the same time period)? Were inclusion and exclusion criteria for being in the study prespecified and applied uniformly to all participants?

Q5. Was a sample size justification, power description, or variance and effect estimates provided?

Q6. For the analyses in this paper, were the exposure(s) of interest measured prior to the outcome(s) being measured?

Q7. Was the timeframe sufficient so that one could reasonably expect to see an association between exposure and outcome if it existed?

Q8. For exposures that can vary in amount or level, did the study examine different levels of the exposure as related to the outcome (eg, categories of exposure, or exposure measured as continuous variable)?

Q9. Were the exposure measures (independent variables) clearly defined, valid, reliable and implemented consistently across all study participants?

Q10. Was the exposure(s) assessed more than once over time?

Q11. Were the outcome measures (dependent variables) clearly defined, valid, reliable and implemented consistently across all study participants?

Q12. Were the outcome assessors blinded to the exposure status of participants?

Q13. Was loss to follow-up after baseline $20 \%$ or less?

Q14. Were key potential confounding variables measured and adjusted statistically for their impact on the relationship between exposure(s) and outcome(s)?

NA, not applicable; NR, not reported.

the USA, ${ }^{29}$ Brazil $^{27}$ and Iraq. ${ }^{22}$ One study did not report on the study location. ${ }^{28}$ The sample size varied from 18 to 1426 among included studies. In one study, working status was not reported. ${ }^{29}$ One study included employed and unemployed participants. ${ }^{30}$ Two studies did not include women, ${ }^{2733}$ and three did not report on sex. ${ }^{22} 2431$

Three studies assessed work stress using the current major measures (job demand-control model and effortreward imbalance model). ${ }^{27} 33{ }^{34}$ Work stress was assessed using the Karasek job strain model, ${ }^{2733}$ the Effort-Reward Imbalance model, ${ }^{34}$ the Brief Job Stress Questionnaire developed by referring to the demand-control-support model in Japan, ${ }^{32}$ a self-reported job stress, ${ }^{24}$ the Occupational Stress Indicator, ${ }^{23}{ }^{28}$ an Occupational Stress Index by Srivastava and Singh, ${ }^{31}$ the Life Events Scale, ${ }^{22}{ }^{30}$ and the Problems of Everyday Living Scale by Pearlin and Schooler. ${ }^{29}$

Three studies presented only descriptive statistics. 229931 Eight studies performed regression analyses ${ }^{232427283032-34}$; but two of the eight studies did not report the types of a regression modelling used. ${ }^{23} 28$ Only two studies sufficiently adjusted for potential confounders such as socioeconomic status and work-related variables. ${ }^{27} 34$

\section{Dental caries and work stress}

One study reported the cross-sectional association between work stress and dental caries, which included 164 paid male workers aged 35-44 years in Brazil. ${ }^{27}$ Work stress was assessed according to the Karasek job strain model. ${ }^{35}$ Dental caries status was assessed using the DMFS index (the number of decayed (D), missing (M) and filled (F) teeth surfaces per person). After adjusting for covariates, one-point increases in the work mental demand, work control, and work variety scores were associated with 0.19 $(95 \% \mathrm{CI}=-0.91$ to 1.29$), 0.87(95 \% \mathrm{CI}=-0.18$ to 1.91$)$, and $-0.06(95 \% \mathrm{CI}=-1.57$ to 1.45$)$ increases in the DMFS index, respectively, in a multivariable regression analysis. Consequently, this study reported a non-significant association between work stress and dental caries. ${ }^{27}$ 


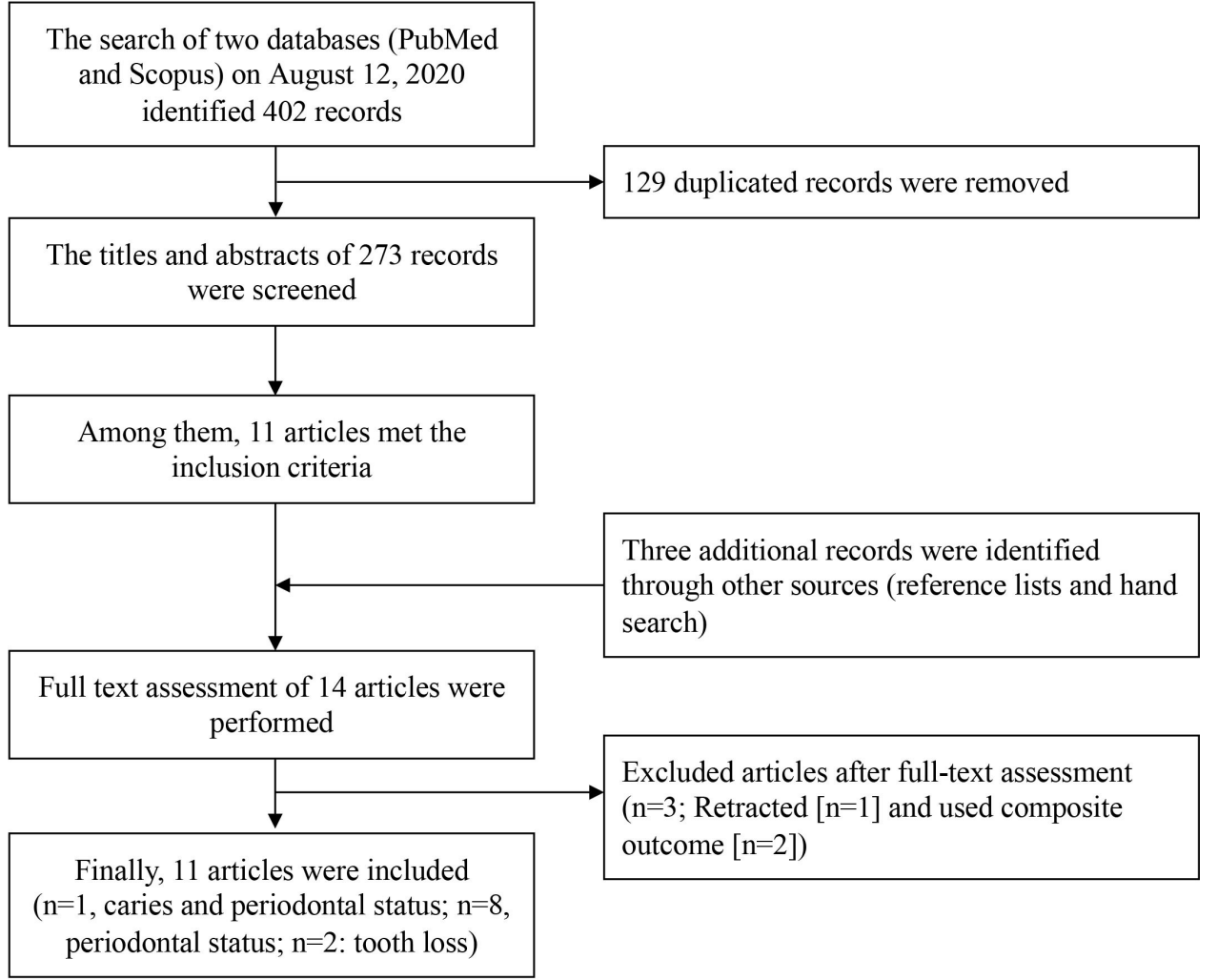

Figure 1 Flow of search strategy and selection of studies for a systematic review.

\section{Periodontal status and work stress}

Eight of nine studies reported a significant association between work stress and worse periodontal status. ${ }^{22-24}$ 27-32 The measurements of periodontal status varied across the included studies. The measurements included probing pocket depth, ${ }^{22} 2331$ clinical attachment level, ${ }^{22} 2829$ alveolar bone loss, ${ }^{29}$ Gingival Index,${ }^{22}$ bleeding on probing, ${ }^{22}$ the Community Periodontal Index and Treatment Needs protocol, ${ }^{24}$ and a composite outcome, including these measures. ${ }^{27}{ }^{32}$ Eight studies assessed periodontal status based on oral examination with probe, but one study was based on only visual inspection by dentists. ${ }^{32}$

Among the nine studies, two studies had unclear methodology; therefore, they were categorised as unknown. ${ }^{2328}$ Freeman and Goss assessed work stress and periodontal status over a 12-month period. ${ }^{23}$ However, they did not clearly report when work stress and periodontal status variables were assessed and how they were used in the statistical models. Linden et al followed up patients for 5.5 years, but work stress was only assessed at the follow-up examination, not at the baseline survey. ${ }^{28}$

Among the remaining seven studies, after excluding the above two studies, three studies presented only descriptive statistics. ${ }^{22} 2931$ The remaining four papers reported significant associations following regression analyses. ${ }^{24} 273032$ However, Akhter et al used general stress questions not specific to work stress and included nonworking adults. ${ }^{30}$ Islam et al used the Brief Job Stress Questionnaire derived from the demand-control-support model in Japan, and periodontal status was assessed based on the visual inspection by dentists. ${ }^{32}$ Important potential confounders, such as socioeconomic status and workrelated variables, were not included. Ramji assessed work stress using a single job stress question and did not adjust for covariates in the statistical models. ${ }^{24}$ Marcenes and Sheiham reported a significant association between periodontal status and work stress. ${ }^{27}$ Periodontal status was assessed by the presence or absence of gums bleeding on probing or with pockets. The authors divided periodontal measures into groups based on 'complete absence of teeth with gums bleeding on probing and with pockets,' or 'the presence of any tooth with gums bleeding on probing or pockets,' and defined the latter as those with periodontal disease. After adjusting for covariates, one-point increases in work mental demand scores, work control scores, and work variety scores were associated with ORs of 1.22 (95\% CI $=1.06$ to 1.37$), 0.97(95 \% \mathrm{CI}=0.88$ to 1.07$)$, and 0.99 (95\% CI=0.85 to 1.16), respectively, for having periodontal disease, in a logistic regression model.

\section{Tooth loss and work stress}

Two studies on the association between work stress and tooth loss were identified. One of the two reported a significant association between work stress and tooth loss. ${ }^{33}$ Hayashi et al reported the association between work stress, assessed using the Karasek job strain model and tooth loss. ${ }^{33}$ A total of 322 male workers employed at a manufacturing company were included. They dichotomised the number of tooth loss into $\leq 3$ and $\geq 4$. After adjusting for covariates, high job demand and low control 
conditions were associated with high odds of having $\geq 4$ teeth loss but not significant $(\mathrm{OR}=1.2 \quad(95 \% \quad \mathrm{CI}=0.40$ to 3.42)). This study did not adjust for the important potential confounders such as socioeconomic status and work-related variables. Sato et al reported the association between work stress, assessed using the effortreward imbalance model and self-reported tooth loss. ${ }^{34}$ After adjusting for covariates including socioeconomic status and work-related variables, a high effort-reward imbalance ratio was significantly associated with a high prevalence of $\geq 1$ tooth loss (prevalence ratio $=1.20(95 \%$ $\mathrm{CI}=1.01$ to 1.42$)$ ).

\section{Study quality}

Table 2 presents the results of the quality assessments for each study. Eight studies $(73 \%)$ had poor quality, while three $(27 \%)$ were rated as fair. None of the studies addressed questions 6 ('For the analyses in this paper, were the exposure(s) of interest measured prior to the outcome(s) being measured?'); 7 ('Was the timeframe sufficient so that one could reasonably expect to see an association between exposure and outcome if it existed?') and 10 ('Was the exposure(s) assessed more than once over time?'), because all the studies were cross-sectional or the study design was unclear.

\section{DISCUSSION}

This is the first systematic review to evaluate and summarise the existing literature on the associations between work stress and oral conditions. As our findings showed, only one study reported on dental caries and periodontal status, nine on periodontal status and two on tooth loss. Based on the findings of this review, the evidence is lacking on the association of work stress with dental caries and tooth loss. Eight of nine studies reported the significant associations between multiple periodontal measures and work stress.

\section{Limitations of the review}

This systematic review has four limitations. First, the systematic literature search, screening and quality assessments were conducted by only one investigator. A single screening could miss more studies than a double screening. ${ }^{36}$ Second, only English-language literature was included. Although a systematic review found no bias due to English-language restriction in systematic reviews, ${ }^{37}$ this review might include bias. Third, there was no protocol for this systematic review. A priori systematic review protocol registration provides the rigour and trustworthiness of the reviews. ${ }^{38}$ This might weaken the rigour and trustworthiness of our review. Finally, a meta-analysis could not be conducted owing to the heterogeneity of the included studies. Work stress was assessed using varied measures. Particularly, only a few studies used the current major measures of work stress. Indicators of periodontal status were also varied. No study used valid epidemiological definitions for periodontal disease as the outcome.
The cut-off points differed between the two studies on tooth loss and work stress. Besides, there was only one study on dental caries and work stress. These limitations hindered us from performing a meta-analysis.

\section{Dental caries and work stress}

We found only one study on the cross-sectional association between work stress and dental caries. ${ }^{27}$ The conclusion was that there was no significant association between work stress and dental caries. However, since the sample size was relatively small $(n=164)$, there is the possibility of a false negative association. Besides, each subscale of the Karasek job strain model was simultaneously included in the statistical model. Generally, in the Karasek job strain model, the recommendation is to use four categories of job strain generated by the interaction of the subscales: high-strain jobs, active jobs, low-strain jobs and passive jobs. ${ }^{9}$ Due to the above treatments of the subscales, it is possible that the association was underestimated. Additionally, as there was no cohort study, we could not assess the prospective associations. Considering the above limitations, it was difficult to determine whether work stress is associated with dental caries. A further study should include a cohort design and a relatively large sample size with appropriate work stress measures.

\section{Periodontal status and work stress}

Nine studies reported on the association between work stress and periodontal status. ${ }^{22-24}$ 27-32 However, the outcome measures were varied across the included studies. Although there are the accepted epidemiological definitions of periodontitis according to the European Workshop in Periodontology and the Centers for Disease Control/American Academy of Periodontology, ${ }^{39} 40$ there was no study that used the definitions. It means that the included studies reported the associations between work stress and periodontal measures, not periodontal disease. In addition, the measurement of work stress measured also varied across studies. Each measure assessed different dimensions of work stress. ${ }^{41}$ Due to the heterogeneity of exposures and outcomes, we could not conduct a meta-analysis.

Of the nine studies, only one study adjusted for the potential confounders, such as socioeconomic status and work-related variables. ${ }^{27}$ Besides, no cohort study was found. The failure to adjust for the confounders and consider the induction time weakens the research evidence. However, despite the above limitations, the consistent association between work stress and worse periodontal status is noteworthy. To verify the current results, a further cohort study using the validated definitions of periodontal disease and current measurements of work stress, in addition to adjusting for the potential confounders, should be performed.

\section{Tooth loss and work stress}

Two studies on the association between work stress and tooth loss were identified. Hayashi $e t$ al s study included 
only male workers employed at one manufacturing company. ${ }^{33}$ In contrast, Sato et $a l$ s study included active workers sampled from a general population. ${ }^{34}$ However, the response rate was relatively low (32\%). The generalisability of both studies could be limited.

The two studies had different cut-off points of tooth loss. Hayashi $e t a l$ s study used the cut-off point of more than four teeth lost. The cut-off point is higher than the mean number of teeth loss (at 25-34, 35-45, 46-54 and 55-64 years $=0.16,0.58,1.48$ and 4.00 , respectively) reported by the national statistical surveys. ${ }^{42}$ This study targeted severe cases only. In Sato et als study, the outcome was the loss of at least more than one tooth. However, this outcome relied on self-reported answers; therefore, selfreported bias might exist.

Both studies showed an increased risk of tooth loss, although only one of the two studies reported a significant association between work stress and tooth loss. However, due to the above limitations, it is difficult to derive any form of conclusion. In the future, a cohort study including general workers should be conducted to confirm these findings.

\section{Conclusions}

Based on the findings, this systematic review suggests a lack of evidence on the association of work stress with dental caries and tooth loss. Although eight of the nine studies reported significant associations between multiple periodontal measures and work stress, no study used valid epidemiological definitions of periodontal disease. For future research, well-designed cohort studies including potential confounding factors and the use of generally accepted measurements of work stress and periodontal disease are needed.

Contributors YSato contributed to the acquisition and the interpretation of data and drafting of the work. YSaijo and EY revised it critically for important intellectual content. All authors contributed to the conception and design of the work, approved the final version to be published, and agreed to be accountable for all aspects of the work in ensuring that questions related to the accuracy or integrity of any part of the work are appropriately investigated and resolved.

Funding This study was supported by Grant-in-Aid for Early-Career Scientists from the Japan Society for the Promotion of Science KAKENHI (grant number JP19K19306)

Competing interests None declared.

Patient consent for publication Not required.

Provenance and peer review Not commissioned; externally peer reviewed.

Data availability statement № data are available. Not applicable.

Supplemental material This content has been supplied by the author(s). It has not been vetted by BMJ Publishing Group Limited (BMJ) and may not have been peer-reviewed. Any opinions or recommendations discussed are solely those of the author(s) and are not endorsed by BMJ. BMJ disclaims all liability and responsibility arising from any reliance placed on the content. Where the content includes any translated material, BMJ does not warrant the accuracy and reliability of the translations (including but not limited to local regulations, clinical guidelines, terminology, drug names and drug dosages), and is not responsible for any error and/or omissions arising from translation and adaptation or otherwise.

Open access This is an open access article distributed in accordance with the Creative Commons Attribution Non Commercial (CC BY-NC 4.0) license, which permits others to distribute, remix, adapt, build upon this work non-commercially, and license their derivative works on different terms, provided the original work is properly cited, appropriate credit is given, any changes made indicated, and the use is non-commercial. See: http://creativecommons.org/licenses/by-nc/4.0/.

\section{ORCID iDs}

Yukihiro Sato http://orcid.org/0000-0001-5657-8379

Yasuaki Saijo http://orcid.org/0000-0002-6211-8202

\section{REFERENCES}

1 GBD 2017 Oral Disorders Collaborators, Bernabe E, Marcenes W, et al. Global, regional, and national levels and trends in burden of oral conditions from 1990 to 2017: a systematic analysis for the global burden of disease 2017 study. J Dent Res 2020;99:362-73.

2 Pitts NB, Zero DT, Marsh PD, et al. Dental caries. Nat Rev Dis Primers 2017:3:17030.

3 Kinane DF, Stathopoulou PG, Papapanou PN. Periodontal diseases. Nat Rev Dis Primers 2017;3:17038.

4 Sheiham A, Croog SH. The psychosocial impact of dental diseases on individuals and communities. J Behav Med 1981;4:257-72.

5 Reisine ST. The impact of dental conditions on social functioning and the quality of life. Annu Rev Public Health 1988;9:1-19.

6 Righolt AJ, Jevdjevic M, Marcenes W, et al. Global-, regional-, and country-level economic impacts of dental diseases in 2015. J Dent Res 2018;97:501-7.

7 Lundberg $\mathrm{U}$, Cooper $\mathrm{C}$. The new workplace in a rapidly changing World. In: The science of occupational health: stress, psychobiology, and the new world of work. Chichester: Wiley-Blackwell, 2010: 11-21.

8 Kivimäki M, Nyberg ST, Batty GD, et al. Job strain as a risk factor for coronary heart disease: a collaborative meta-analysis of individual participant data. Lancet 2012;380:1491-7.

9 Berkman L, Kawachi I, Theorell T. Working conditions and health. In: Social epidemiology. New York: Oxford University Press, 2014: 153-81.

10 Lundberg U, Cooper C. Stress-related health problems. In: The science of occupational health: stress, psychobiology, and the new world of work. Chichester: Wiley-Blackwell, 2010: 75-91.

11 Béjean S, Sultan-Taïeb H. Modeling the economic burden of diseases imputable to stress at work. Eur J Health Econ 2005;6:16-23.

12 Sabbah W, Gomaa N, Gireesh A. Stress, allostatic load, and periodontal diseases. Periodontol 2000 2018;78:154-61.

13 Gomaa N, Glogauer M, Tenenbaum H, et al. Social-biological interactions in oral disease: a 'cells to society' view. PLoS One 2016;11:e0146218.

14 Tikhonova S, Booij L, D'Souza V, et al. Investigating the association between stress, saliva and dental caries: a scoping review. BMC Oral Health 2018;18:41.

15 Castro MML, Ferreira RdeO, Fagundes NCF, et al. Association between psychological stress and periodontitis: a systematic review. Eur J Dent 2020;14:171-9.

16 Lundberg U, Cooper C. Introduction: History of work and health. In: The science of occupational health: stress, psychobiology, and the new world of work. Chichester: Wiley-Blackwell, 2010: 1-10.

17 Moher D, Liberati A, Tetzlaff J, et al. Preferred reporting items for systematic reviews and meta-analyses: the PRISMA statement. BMJ 2009;339:b2535.

18 Liberati A, Altman DG, Tetzlaff J, et al. The PRISMA statement for reporting systematic reviews and meta-analyses of studies that evaluate healthcare interventions: explanation and elaboration. $B M J$ 2009;339:b2700.

19 Dekkers OM, Vandenbroucke JP, Cevallos M, et al. COSMOS-E: guidance on conducting systematic reviews and meta-analyses of observational studies of etiology. PLoS Med 2019;16:e1002742.

20 Stroup DF, Berlin JA, Morton SC. Meta-analysis of observational studies in epidemiology: a proposal for reporting. JAMA 2000;283:2008-12.

21 National Institutes of Health. Quality assessment tool for observational cohort and cross-sectional studies, 2014. Available: https://www.nhlbi.nih.gov/health-topics/study-quality-assessmenttools [Accessed 17 Sep 2020]

22 Talib Bandar K. The association between periodontal disease and job stress in Baghdad City. J Kerbala Univ 2009;5:47-57.

23 Freeman R, Goss S. Stress measures as predictors of periodontal disease - a preliminary communication. Community Dent Oral Epidemiol 1993;21:176-7.

24 Ramji R. Assessing the relationship between occupational stress and periodontitis in industrial workers. PHD thesis, Umeå international school of public health 2011. 
25 Yoshino K, Suzuki S, Ishizuka Y, et al. Relationship between job stress and subjective oral health symptoms in male financial workers in Japan. Ind Health 2017;55:119-26.

26 Capurro DA, Davidsen M. Socioeconomic inequalities in dental health among middle-aged adults and the role of behavioral and psychosocial factors: evidence from the Spanish National Health Survey. Int J Equity Health 2017;16:34.

27 Marcenes WS, Sheiham A. The relationship between work stress and oral health status. Soc Sci Med 1992;35:1511-20.

28 Linden GJ, Mullally BH, Freeman R. Stress and the progression of periodontal disease. J Clin Periodontol 1996;23:675-80.

29 Genco RJ, Ho AW, Grossi SG, et al. Relationship of stress, distress and inadequate coping behaviors to periodontal disease. $J$ Periodontol 1999;70:711-23.

30 Akhter R, Hannan MA, Okhubo R, et al. Relationship between stress factor and periodontal disease in a rural area population in Japan. Eur J Med Res 2005;10:352-7.

31 Mahendra L, Mahendra J, Austin RD. Stress as an aggravating factor for periodontal diseases. J Clin Diagn Res 2011;5:889-93.

32 Islam MM, Ekuni D, Yoneda T, et al. Influence of occupational stress and coping style on periodontitis among Japanese workers: a crosssectional study. Int J Environ Res Public Health 2019;16:3540.

33 Hayasht N, Tamagawa H, Tanaka M, et al. Association of tooth loss with psychosocial factors in male Japanese employees. J Occup Health 2001;43:351-5.

34 Sato Y, Tsuboya T, Aida J, et al. Effort-reward imbalance at work and tooth loss: a cross-sectional study from the J-SHINE project. Ind Health 2020;58:26-34.
35 Karasek RA. Job demands, job decision latitude, and mental strain: implications for job redesign. Adm Sci Q 1979;24:285-308.

36 Waffenschmidt S, Knelangen M, Sieben W, et al. Single screening versus conventional double screening for study selection in systematic reviews: a methodological systematic review. BMC Med Res Methodol 2019;19:132.

37 Morrison A, Polisena J, Husereau D, et al. The effect of Englishlanguage restriction on systematic review-based meta-analyses: a systematic review of empirical studies. Int J Technol Assess Health Care 2012;28:138-44.

38 Shamseer L, Moher D, Clarke M, et al. Preferred reporting items for systematic review and meta-analysis protocols (PRISMA-P) 2015 elaboration and explanation. BMJ 2015;349:g7647.

39 Page RC, Eke PI. Case definitions for use in population-based surveillance of periodontitis. J Periodontol 2007;78:1387-99.

40 Tonetti MS, Claffey N, European Workshop in Periodontology group $\mathrm{C}$. Advances in the progression of periodontitis and proposal of definitions of a periodontitis case and disease progression for use in risk factor research. group $\mathrm{C}$ consensus report of the 5th European workshop in Periodontology. J Clin Periodontol 2005;32:210-3.

41 Leka S, Jain A, World Health Organization. Health impact of psychosocial hazards at work: an overview, 2010. Available: https:// apps.who.int/iris/handle/10665/44428

42 Ministry of Health, Labour and Welfare (Japan). Dental diseases survey, 2016. Available: https://www.mhlw.go.jp/toukei/list/62-17b. html [Accessed 12 Mar 2021]. 